\title{
Simulación de la dispersión de contaminantes del agua de la cuenca del Río Huaura
} Simulation the dispersion of water pollutants from the Huaura River basin

\author{
Edith Meryluz Claros Guerrero ${ }^{1}$, Jaqueline Jessica Cabello Blanco ${ }^{1}$, Johnny Gregorio \\ Cipriano Bautista ${ }^{1}$
}

\section{RESUMEN}

Objetivo: Determinar un modelo matemático que permita conocer la dispersión de contaminantes del agua de la Cuenca del Río Huaura en el tramo Estación Alco - Desembocadura de Carquín. Métodos: Esta investigación se realizó en el periodo transicional entre avenida a estiaje (setiembre - diciembre), identificándose el tramo del rio con flujo uniforme $(3+800 \mathrm{~km})$. Se usó la ecuación de advección - difusión unidimensional con coeficiente constante, mediante diferencias finitas, para simular la dispersión del soluto (colorante alimentario), cuyos datos se obtuvieron mediante inyección instantánea de corta duración de un trazador en el río, tomándose muestras cada 2,5 metros río abajo en el tramo de estudio y evaluándose la dispersión mediante la valoración de saturación del color de la muestra de agua. Resultados: Se observó que el soluto se dispersa en la medida que se desplaza el fluido. Se ajustó a la ecuación de advección - difusión unidimensional con coeficiente constante con un error medio de 0,44998. Conclusiones: Si se cuenta con un fluido laminar con mínima turbulencia da como resultado un buen ajuste los datos experimentales con los datos simulados, asumiendo valores constantes de la velocidad promedio del fluido y dispersión longitudinal.

Palabras clave: Dispersión de contaminante, Río Huaura, trazador, ecuación de advección.

\begin{abstract}
Objective: to determine a mathematical model that allows to know the dispersion of pollutants from the water of the Huaura River Basin in the section Estación Alco - Mouth of Carquín. Methods: this investigation was carried out during the period of transition of river flood and low waters (September to December), identifying the section of the river with uniform flow $(3+800 \mathrm{~km})$, the advection diffusion equation was used one-dimensional with constant coefficient, and by means of finite differences, to simulate the dispersion of the solute (food coloring), whose data were obtained by instantaneous injection of a short-term tracer in the river, taking samples every 2.5 meters downstream in the section of the study and evaluating the dispersion by saturation of water color. Results: It was observed that the solute is dispersed as the fluid moves, it was adjusted to the advection equation - one-dimensional diffusion with constant coefficient with an average error of 0.44998. Conclusions: If there is a laminar fluid with minimum turbulence, the experimental data with the simulated data are adjusted correctly, assuming constant values of the average fluid velocity and longitudinal dispersion.
\end{abstract}

Keywords: pollutant dispersion, Huaura River, tracer, advection equation.

\footnotetext{
${ }^{1}$ Universidad Nacional José Faustino Sánchez Carrión, Huacho, Perú.
} 


\section{INTRODUCCIÓN}

El crecimiento de la población, las actividades agrícolas, ganaderas y el desarrollo industrial son las causas principales del aporte de residuos y efluentes que contaminan las aguas subterráneas, ríos, lagos y mares, modificando el hábitat natural de la fauna y flora, generando desequilibrio en el ecosistema destruyéndose la armonía entre el hombre y su medio.

Cuando se vierte una sustancia contaminante al medio ambiente, ésta no permanece en el lugar donde se realiza el vertido, sino que se produce el proceso de transporte, que pueden dividirse en dos categorías: advección y dispersión. La advección se refiere al traslado del contaminante a favor de la corriente del medio de transporte si se vacía una bolsa de tintura en el centro de un río, la advección llevará la mancha resultante río abajo, mientras la dispersión se refiere al transporte del compuesto a través de la acción de movimientos aleatorios (Mihelcic y Zimmerman, 2014).

La utilización de modelos matemáticos para simular los procesos de transporte y dispersión de los contaminantes vertidos en medios receptores, ha experimentado un auge en las últimas décadas. Esto debido a la necesidad de estudiar o evaluar la contaminación generada por las aguas vertidas y su impacto en el medio receptor, ya que afecta el desarrollo de las actividades humanas y, en general, la calidad del medio ambiente, de esta forma la modelación matemática de la calidad del agua se ha convertido en una herramienta que contribuye a la planificación integral de los recursos hídricos (Torres- Bejarano et al., 2015).

La modelación de transporte de contaminantes en ríos requiere el conocimiento de parámetros hidrológicos (caudal, velocidad, coeficiente de dispersión) y geométricos (profundidad, anchura, sección transversal, pendiente) (Monerris, 1995). Yzocupe (2005), en su investigación "Modelo de dispersión de contaminantes en aguas rasas", presenta un modelo de dispersión de contaminantes bidimensionales construido sobre la base de un modelo hidrodinámico. El modelo acoplado se basa en la aproximación numérica de las ecuaciones bidimensionales que rigen la dinámica de las aguas rasas y la dispersión de sustancias. Tales ecuaciones son las de continuidad del cuerpo de fluido, de movimientos en las direcciones $\mathrm{X}$ e $\mathrm{Y}, \mathrm{y}$ de conservación de la concentración de una sustancia diluida. Las variables dependientes son las velocidades medias $\mathrm{U}$ y $\mathrm{V}$ en las direcciones $X$ e $Y$ respectivamente; el nivel $n$ del agua con respecto al plano de referencia y la concentración $\mathrm{C}$ de la sustancia diluida. La aproximación numérica está basada en el esquema de diferencias finitas explícito de Fischer. El modelo cuenta con las condiciones iniciales y de fronteras adecuadas.

Quispe et al. (2016) en su investigación de dispersión de contaminantes en cursos de agua en el río Chumbao, Perú, estudia el modo de dispersión de un contaminante vertido en un curso de agua mediante un método experimental consistente en el uso de un "marcador" o sustancia "traza", aplicado en un tramo de 100 metros del río Chumbao en la Provincia de Andahuaylas, Perú, cuyo marcador utilizado es una solución de sal común (cloruro de sodio) vertido mediante un tubo de dos pulgadas, recolectando las muestras tramo abajo cada 30 segundos, analizando la conductividad eléctrica de las muestras se estima la concentración de marcador en cada punto de recolección, y con ello los posibles valores de los parámetros de dispersión de un eventual contaminante sobre el río y comprueba que un modelo simple de dispersión gaussiana representa de un modo suficientemente preciso las condiciones observadas.

El método de solución de expresiones analíticas a partir del análisis del balance de masa por advección neta versus difusión transversal para flujo uniforme es un método que determina la dispersión longitudinal; mientras el método de trazadores es el 
método más preciso, aunque requiere mayor trabajo de campo y laboratorio (Fischer et al., 1979). Por otro lado en la situación de una evaluación preliminar de contaminación o en ausencia de datos de campo, el coeficiente de dispersión puede ser estimado en base a expresiones empíricas publicadas en la literatura (Menéndez, 2010).

El trazador es una sustancia específica que sirve para "marcar" o hacer más fácilmente identificable una fase específica o parte de un sistema denominado material marcado. Espinoza et al. (2013) menciona que un trazador debe cumplir como mínimo dos requisitos fundamentales: comportarse similar o igual al material marcado, y tener por lo menos una propiedad que distinga del material marcado, de forma tal que sea fácilmente detectable en presencia de otros materiales. Por ende un trazador ideal debe cumplir entre otras condiciones de fácil detección cualitativa y cuantitativa, ausente en las aguas naturales o que se presente en bajas concentraciones, no toxicidad hacia los seres vivos y disponibilidad económica y presencia en el mercado.

La cuenca del río Huaura está localizada en la costa central del Perú, y pertenece al sistema hidrográfico de la vertiente del océano pacífico de la Cordillera de los Andes, con un área de drenaje total, hasta su desembocadura en el océano pacífico de $4392 \mathrm{~km}^{2}$, tiene sus orígenes en la Cordillera de Raura a una altitud que varía entre 4500 a $5600 \mathrm{msnm}$ discurre en relación NE - SO hacia el distrito de Sayán y después deriva hacia el oeste para desembocar en el mar en el distrito de Caleta Carquín. Entre los parámetros de calidad determinados por los estándares de calidad ambiental, se tiene el pH (número de iones de hidrógeno presentes que determinan el carácter ácido o básico del agua) y la temperatura (que determina las condiciones para muchas reacciones químicas y procesos biológicos). Según las características climatológicas, la cuenca de río Huaura tiene descargas máximas de enero a marzo y descargas mínimas de julio a setiembre, identificándose tres periodos dentro del ciclo anual: periodo de avenidas, de estiaje y un periodo transicional entre avenidas y estiaje, lo que implica la variación del caudal del Río Huaura.

La presente investigación tuvo como objetivo desarrollar un modelo matemático que permita conocer la dispersión de contaminantes del agua de la Cuenca del Río Huaura en un tramo de la Estación Alco Desembocadura de Carquín.

\section{MATERIALES Y MÉTODOS}

Esta investigación se desarrolló en el periodo transicional entre avenida y estiaje correspondiente a los meses de setiembre a diciembre del 2017, eligiéndose el tramo $3+800 \mathrm{Km}$ a 300 metros del Puente de Huaura en la influencia del distrito de Huaura, que corresponde al tramo de la Estación Alco Desembocadura de Carquín, que por los trabajos de descolmatación y encauzamiento del río realizado por las autoridades regionales y locales, se adaptó a los propósitos de la investigación, considerándose como área de estudio de 40 metros de longitud, el cual se eligió basado en las recomendaciones realizadas por Quispe et al. ( 2016), quien manifiesta que el tramo del río para realizar el estudio debe tener las siguientes características: Estable, libre de rocas, plantas y otras obstrucciones que puedan crear turbulencia. Lecho plano, uniforme y estable para reducir componentes verticales de velocidad.

Para la determinación del modelo de dispersión de contaminantes, se usó la ecuación de advección difusión unidimensional con coeficientes constantes, y fue necesario conocer los parámetros hidrológicos y geométricos acordes a la naturaleza del área de estudio; evaluándose los parámetros hidrológicos: caudal, velocidad, coeficiente de dispersión; geométricos: profundidad, anchura, sección transversal, utilizándose el método de flotadores, recomendado por el Senamhi 
(Chamorro, 2011), procediéndose a demarcar a lo largo del área de estudio, cuatro secciones de igual longitud $(10 \mathrm{~m})$, midiéndose en cada sección la anchura del río y profundidad del fluido; para determinar la velocidad se registró el tiempo empleado por el flotador en recorrer cada tramo, esta actividad fue repetida 10 veces; y se obtuvieron los resultados siguientes: ancho del río de 6,40 m, velocidad superficial de 1,06 $\mathrm{m} / \mathrm{s}$ profundidad promedio de $26 \mathrm{~cm}$, área transversal de $1,64 \mathrm{~m}^{2}$ y caudal de $\mathrm{Q}=1,477 \mathrm{~m}^{2} / \mathrm{s}$

Para medir la dispersión de contaminantes se optó por el método de trazadores, para ello se utilizó colorantes artificiales (rojo y azul) y se preparó la solución en 3 litros de agua del mismo río, considerando colorantes artificiales E-129, y E-132 con una solubilidad de $10 \mathrm{~g} / \mathrm{L}$ (soluto), como trazador, evaluando el color de la concentración mediante la aplicación color grab, luego se vertió la solución en el centro de la longitud transversal del río, se tomó la temperatura y se midió el pH con un pH-metro, observándose que no existió variación del $\mathrm{pH}$ del agua, con o sin colorante; usando la técnica de inyección instantánea de corta duración y tomándose muestras de agua: previas a la inyección del trazador, en la mezcla de soluto y luego de adicionar la mezcla en el río; se recogió muestras de agua, en tramos de igual longitud (2,5 $\mathrm{m})$ río abajo a lo largo del área de estudio; y al no contar con equipos electrónicos sofisticados se optó por evaluar in situ el color de la concentración (saturación del color y la variación del mismo), según el modelo $(\mathrm{H}, \mathrm{S}, \mathrm{V})$ que corresponde a matiz, saturación del color (0 a 100), y brillo (0 a 100). Para 30 gramos del colorante (soluto) en tres litros de agua, antes de la inyección se obtuvo el porcentaje de saturación del color del color de $\mathrm{H}=210, \mathrm{~S}=$ 67 y $\mathrm{V}=13$, con temperatura del agua de $26,1^{\circ} \mathrm{C}$ y $\mathrm{PH}$ de 8,46
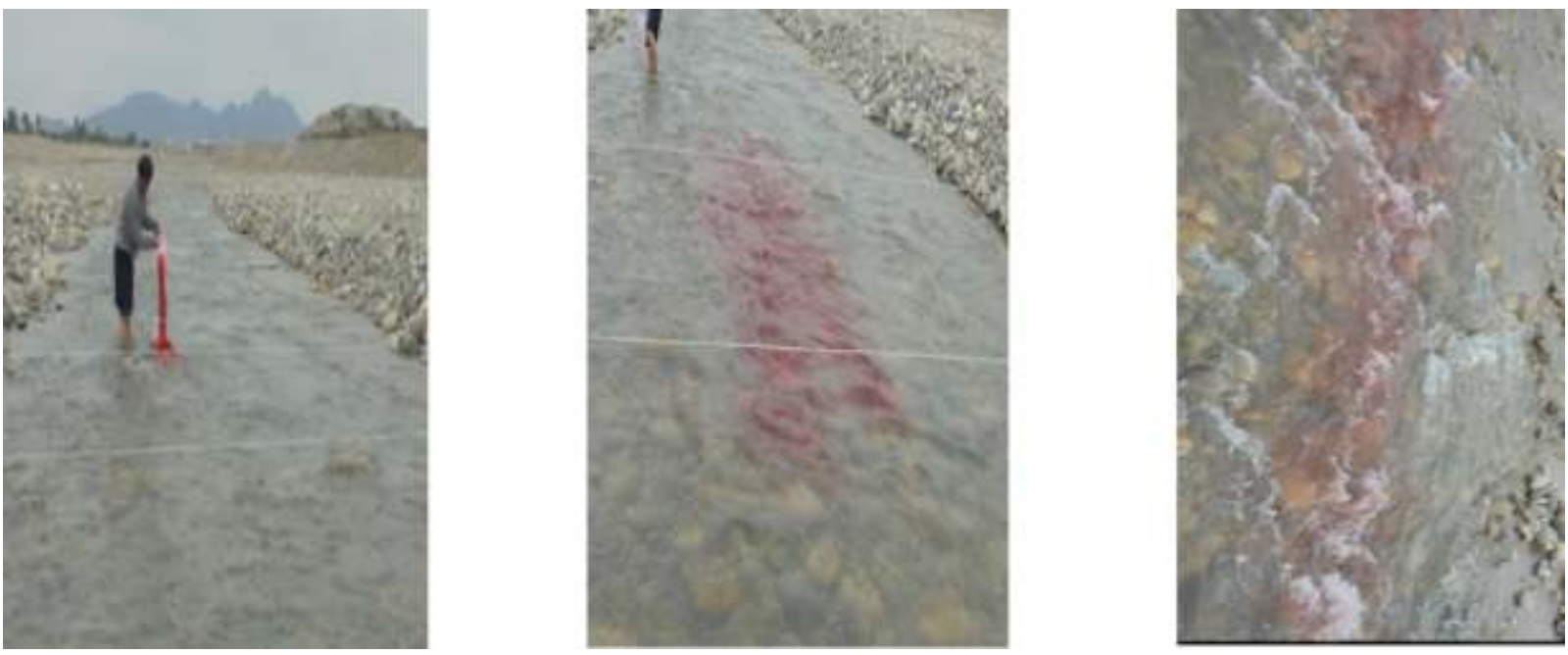

Figura 1. (a) vertido del soluto en el agua (b) y (c) desplazamiento del colorante por las secciones a analizar. 
Para simular la dispersión de contaminantes (soluto), es decir la contaminación del curso por una sustancia en cualquier punto $Z$ puede representarse por su valor promedio $c$ sobre toda la sección transversal $\mathrm{A}$. Si, además, se considera que se trata de una sustancia conservativa (no sujeta a reacciones internas) y que no existen intercambios con el medio, es decir, que solo actúan el transporte advectivo y el difusivo, la ecuación de transporte es la ecuación diferencial de segundo orden no homogéneo (1) que nos permite describir una figura tridimensional (modelo matemático de Sing y Beck), esta ecuación asume valores constantes para $v$ y $D$, mezcla uniforme a través de la sección y densidades iguales, tanto para el agua como para el soluto.

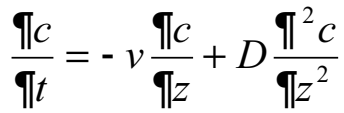

Dónde:
A: Área transversal del río $\left(\mathrm{m}^{2}\right)$
c: Concentración del soluto o contaminante $\left(\mathrm{kg} / \mathrm{m}^{3}\right)$.
Q: Caudal del río $\left(\mathrm{m}^{3} / \mathrm{s}\right)$
Velocidad promedio del fluido: $v=Q / A$
D: Coeficiente de dispersión longitudinal $\left(\mathrm{m}^{2} / \mathrm{s}\right)$

Para la obtención del coeficiente de dispersión longitudinal (D) para ríos y esteros, optamos

por el propuesto por Fischer et al (1979) quien desarrolló la siguiente fórmula:

$$
D=\frac{0,011}{H U^{*}} U^{2} B^{2}
$$

Dónde:

D: Coeficiente de dispersión longitudinal $\left(\mathrm{m}^{2} / \mathrm{s}\right)$

$\mathrm{U}$ : velocidad de la corriente de agua $(\mathrm{m} / \mathrm{s})$

B: Ancho del cauce $(\mathrm{m})$

$\mathrm{H}$ : Profundidad media de la corriente de agua

Velocidad de cizalle: $U^{*}=\sqrt{\mathrm{gHS}} \quad(\mathrm{m} / \mathrm{s})$

g: Aceleración de la gravedad $\left(\mathrm{m} / \mathrm{s}^{2}\right)$

$\mathrm{S}$ : Pendiente longitudinal del cauce (adimensional)

A continuación se procedió a resolver la ecuación diferencial (1), definiéndose la denotó: $c=c\left(z_{i}, t_{j}\right)=c_{i}^{j} ;$ Usando método de concentración del soluto: $c=c(z, t)$; la cual se diferencias finitas:

$$
f^{\prime}(x)=\frac{f\left(x_{i+1}\right)-f\left(x_{i}\right)}{h} \text { y } f^{\prime \prime}(x)=\frac{f\left(x_{i+1}\right)-2 f\left(x_{i}\right)+f\left(x_{i-1}\right)}{h^{2}}
$$

En el punto $\left(\mathrm{z}_{\mathrm{i}}, \mathrm{t}_{\mathrm{j}}\right)$, y la ecuación (1) se obtiene:

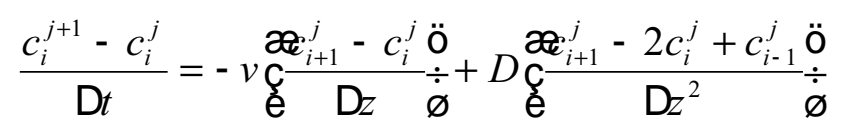


Dado que conocemos el estado en el tiempo j, definimos el estado en el tiempo $j+1$, para esto

despejamos $\mathrm{c}_{\mathrm{i}}^{(\mathrm{j}+1)}$ :

Hacemos: $c_{i}^{j+1}=c_{i}^{j}-\frac{\mathrm{D} t \cdot v}{\mathrm{D} z}\left(c_{i}^{j+1}-c_{i}^{j}\right)+\frac{\mathrm{D} t \cdot D}{\mathrm{D} z^{2}}\left(c_{i+1}^{j}-2 c_{i}^{j}+c_{i-1}^{j}\right)$

$$
\mathrm{g}=\frac{\mathrm{D} t \cdot v}{\mathrm{D} z} \quad \mathrm{t}=\frac{\mathrm{D} t \cdot D}{\mathrm{D} z^{2}}
$$

Obteniéndose la ecuación (2), que nos en cualquier punto y tiempo de la sección permitió conocer la concentración del soluto transversal del rio (Ver tabla 2):

$$
c_{i}^{j+1}=c_{i}^{j}-g\left(c_{i}^{j+1}-c_{i}^{j}\right)+\mathrm{t}\left(c_{i+1}^{j}-2 c_{i}^{j}+c_{i-1}^{j}\right)
$$

Así para calcular $\mathrm{c}_{\mathrm{i}}^{(\mathrm{j}+1)}$ se necesita:.$c_{i-1}^{j}$

$\begin{array}{ll}. c_{i}^{j} & . c_{i}^{j+1} \\ c_{i+1}^{j} & \end{array}$

Y los datos de : $\mathrm{Q}\left(\mathrm{m}^{3} / \mathrm{s}\right), \mathrm{A}\left(\mathrm{m}^{2}\right), \mathrm{D}\left(\mathrm{m}^{2} / \mathrm{s}\right)$, $\Delta \mathrm{t}(\mathrm{s}), \Delta \mathrm{z}(\mathrm{m})$, así como la condición inicial (concentración del soluto ) $\left(\mathrm{kg} / \mathrm{m}^{3}\right)$, los cuales se ingresaron como condiciones iniciales en la hoja de cálculo de Excel para simular los resultados de la dispersión de la concentración del soluto, en tiempo indefinido.

\section{RESULTADOS}

La Tabla 1 muestra la dispersión de la concentración del soluto utilizado en el tramo $3+800$ km del Río Huaura. Se observó la matiz y saturación del color (modelo HSB) de la concentración inicial del soluto de $30 \mathrm{~g} / \mathrm{L}$ al instante de la inyección del soluto en el río, se observó la variación de la matiz y saturación del color de la mezcla correspondiendo a una concentración aproximada de $21 \mathrm{~g} / \mathrm{L}$; disminuyendo aproximadamente a $13,9 \mathrm{~g} / \mathrm{L}$ a una distancia de 10 metros rio abajo; $8,1 \mathrm{~g} / \mathrm{L}$, a los 20 metros y $2,7 \mathrm{~g} / \mathrm{La}$ los 30 metros de distancia recorrida rio abajo desde el inicio de la inyección del soluto. (Tabla 1). 
Tabla 1. Dispersión de la concentración del soluto, en el tramo 3+800 km de la cuenca del río Huaura, tramo Estación Alco-Desembocadura de Carquín (setiembre-diciembre 2017)

\begin{tabular}{|c|c|c|c|c|c|}
\hline \multirow{2}{*}{$\begin{array}{l}\text { Tramo } \\
\text { in }\end{array}$} & \multicolumn{3}{|c|}{ MODELO HSB } & \multicolumn{2}{|c|}{ concentración equivalente } \\
\hline & u & $S$ & V & $g / L$ & $\mathrm{Kg} / \mathrm{m}^{3}$ \\
\hline inicial & 210 & 67 & 13 & 30 & 3 \\
\hline al instante & 234 & 47 & 92 & 21,0 & 2,1 \\
\hline 2,5 & 214 & 80 & 72 & 17,8 & 1,8 \\
\hline 5 & 220 & 99 & 65 & 24,6 & 2,5 \\
\hline 7,5 & 203 & 100 & 78 & $21: 4$ & 2,1 \\
\hline 10 & 197 & 60 & 78 & 13,9 & 1,4 \\
\hline 12,5 & 196 & 61 & 80 & 7,6 & 0,8 \\
\hline 17,5 & 191 & 40 & 68 & 9,4 & 0,9 \\
\hline 20 & 170 & 18 & 79 & 8,1 & 0,8 \\
\hline 22,5 & 204 & 17 & 75 & 7,6 & 0,8 \\
\hline 27,5 & 204 & 5 & 78 & 2,2 & 0,2 \\
\hline 30 & 120 & 6 & 65 & 2,7 & 0,3 \\
\hline
\end{tabular}

Utilizando la ecuación numérica (2), obtenemos la simulación de la concentración del soluto en tiempo indefinido y para medir la distancia entre la curva y los datos estimados usamos el error medio:

$$
E_{1}(c)=\frac{1}{N} \stackrel{\circ}{a=1}_{k=1}^{N}\left|c-c^{*}\right| \text { Burden y Faires, (2002). }
$$

La Tabla 2 muestra la comparación de datos obtenidos de la concentración del soluto in situ (dato real) y los valores estimados por el modelo matemático de nuestro estudio: ecuación (2). Siendo el valor inicial de la concentración de $2,1 \mathrm{~kg} / \mathrm{m}^{3}$ (al instante de la inyección del soluto en el río) dato real considerado como condición inicial del modelo matemático, con un error de 0 ; para $1,8 \mathrm{~kg} / \mathrm{m}^{3}$ se obtiene valor estimado de 2,09998 con un error de 0,29998 con una toleración de $10^{-6} \quad\left(\mathrm{TOL}=10^{-6}\right.$ y así sucesivamente. (Tabla 2 ).

Tabla 2. Comparación de resultados y estimados

\begin{tabular}{rrr|}
\hline Dato real & estimados & $\left|c-c^{\times}\right|$ \\
\hline 2,1 & 2,1 & 0 \\
1,8 & 2,09998 & 0,39998 \\
\hline 2,5 & 2,10003 & 0,39997 \\
2,1 & 2,10026 & 0,00026 \\
1,4 & 2,09965 & 0,69965 \\
0,8 & 2,10003 & 1,30003 \\
& total & 2,69988
\end{tabular}

Luego el error medio es: $E_{1}(f)=\frac{2,69988}{6}=0,44998$ 
Donde $\mathrm{E}_{1}(\mathrm{f})$, es el promedio de los errores de medición que mide la distancia entre la curva y los datos estimados y nos permite determinar la estabilidad del método de ajuste. En nuestro estudio para una $\mathrm{TOL}=10^{-6}$ se obtiene un error medio de 0,44998.

\section{DISCUSIÓN}

Espinoza et al. (2013) mencionan que un trazador debe cumplir como mínimo dos requisitos fundamentales: comportarse similar o igual al material marcado, y tener por lo menos una propiedad que distinga del material marcado, siendo esto muy necesario cuando no se cuenta con equipos electrónicos. Así para esta investigación se utilizó colorantes artificiales (rojo y azul), observándose que no existió variación del pH del agua, con o sin colorante.

Quispe et al. (2016) en su artículo dispersión de contaminantes en curso de agua: estudios en el río Chumbao, Perú, considera que se comprueba que un modelo simple de dispersión gaussiana representa de un modo suficientemente preciso las condiciones observadas, y para la obtención de los posibles valores de los parámetros de dispersión recolectaron muestras cada 30 segundos, utilizando como marcador cloruro de sodio. En esta investigación se usó un colorante alimentario evaluándose la saturación del color, tomando muestras cada 2,5 metros de longitud río abajo, obteniéndose un estimado de la dispersión de contaminantes en el río Huaura, haciendo uso de diferencias finitas, simulándose en una hoja de cálculo de Excel cuando el tiempo tiende al infinito considerándose un buen ajuste que describe la dispersión de contaminantes en el río Huaura.

Por lo que se concluye que la evaluación de la dispersión de contaminantes en una sección del río se ve afectado por las características del fluido; si se cuenta con un fluido laminar con mínima turbulencia la simulación mediante la ecuación de advección y difusión da como resultado un buen ajuste para un caudal relativamente bajo, asumiendo valores constantes de la velocidad promedio del fluido y la dispersión longitudinal; es importante tener mayor número de datos experimentales para obtener una mejor precisión en la simulación de la ecuación advección y difusión, siempre evaluando que la composición química del trazador en su interacción con el fluido sea de fácil detección cualitativa y cuantitativa; las evaluaciones de la dispersión de contaminantes en agua superficiales permitirá conocer los parámetros hidrológicos y geométricos acorde a las características de la zona de estudio de tal manera que se pueda realizar programas de planificación de recursos hídricos, en prevención a la ocurrencia de algún evento no deseado para proceder a adoptar las medidas correctivas de mitigación y prevención de accidentes de mayor envergadura que generará pérdidas económicas y del hábitat afectando el ecosistema del área de impacto, por ende la calidad del medio ambiente. 


\section{REFERENCIAS BIBLIOGRÁFICAS}

Burden, R. y Faires, J. (2002). Análisis Numérico. México: International Thomson Ed. Fischer, et al (1979). Mixing in Inland and Coastal Waters, Academic Press.

Chamorro, G. (2011). Guía de Hidrometría: Estimación del caudal por el método de flotadores. Servicio Nacional de Meteorología e Hidrología del Perú Senamhi.

Espinoza R. et al (2013). Caracterización Hidrodinamica y Dispersión de Contaminantes de la Parte Alta del Río Suquiapa, Santa Ana. Facultad de Ingeniería y Arquitectura, Universidad del Salvador. San Salvador.

Yzocupe, V. (2005). Modelo de dispersión de contaminantes en aguas rasas. Facultad de Ciencias Físicas, Universidad Nacional Mayor de la San Marcos. Lima.

Monerris, M., Marzal, P. (1995), Modelación de la Calidad de Agua. Universidad Politécnica de Valencia.
Menéndez, A. (2010). Transporte de contaminantes en medio acuático. Universidad Tecnológica Nacional.

Mihelcic, J., Zimmerman, J. (2014). Ingeniería a mbiental: fundamentos, sustentabilidad, diseño, México: Alfaomega Grupo Editor, S.A. de C.V.

Torres - Bejarano, F., Ramírez - León, H., Rodríguez, C., Tejera, M. y Vásquez, M. (2015). Validación de un modelo hidrodinámico y calidad del agua para el río Magdalena, en el tramo adyacente a Barranquilla, Colombia.

Quispe Prado, W. et al. (2016). Dispersión de contaminantes en cursos de agua: estudios en el río Chumbao, Perú.

\section{Correo electrónico:}

meryluzclaros@gmail.com

Revisión de Pares:

Recibido: 26-04-2018

Aceptado: 11-06-2018 